\title{
The Impact of The Initial Surface Reconstruction on Heteroepitaxial Film Growth and Defect Formation
}

Jessica E. Bickel

Cleveland State University, j.e.bickel@csuohio.edu

Joanna Mirecki Millunchick

University of Michigan

Follow this and additional works at: https://engagedscholarship.csuohio.edu/sciphysics_facpub

Part of the Physics Commons

How does access to this work benefit you? Let us know!

Publisher's Statement

This is an author-created, un-copyedited version of an article accepted for publication in Physica Scripta. The publisher is not responsible for any errors or omissions in this version of the manuscript or any version derived from it. The Version of Record is available online at http://dx.doi.org/10.1088/0031-8949/89/7/075707

\section{Repository Citation}

Bickel, Jessica E. and Millunchick, Joanna Mirecki, "The Impact of The Initial Surface Reconstruction on Heteroepitaxial Film Growth and Defect Formation" (2014). Physics Faculty Publications. 195.

https://engagedscholarship.csuohio.edu/sciphysics_facpub/195

This Article is brought to you for free and open access by the Physics Department at EngagedScholarship@CSU. It has been accepted for inclusion in Physics Faculty Publications by an authorized administrator of

EngagedScholarship@CSU. For more information, please contact library.es@csuohio.edu. 


\title{
The impact of the initial surface reconstruction on heteroepitaxial film growth and defect formation
}

\author{
J E Bickel and J Mirecki Millunchick
}

\begin{abstract}
While it is well known that growth conditions such as temperature greatly affect defect incorporation in thin films, less is known about the direct effects of the surface reconstruction. In this work, we examine the effect of the initial surface reconstruction on defect incorporation in $\mathrm{GaSb} / \mathrm{GaAs}(001)$ lattice mismatched films. The stress built up in GaSb films grown on As-terminated and Sb-terminated GaAs was monitored during film growth and shows that the total relaxation is similar in both films along the [110], but lower on the Sb-terminated surface along the [110]. These differences can be understood by examining the ability for the two surface terminations to accommodate strain. The resulting films show that the density of 3D islands is lower for the Sb-terminated surfaces, and that lattice mismatch strain is further accommodated by a $5^{\circ}$ tilt. In contrast, the As-terminated surface contains both stacking faults and misfit dislocations. These results demonstrate the possibility to engineer specific defects into films by controlling the starting surface of film growth.
\end{abstract}

Keywords: molecular beam epitaxy, stresses, defects, semiconducting III V materials

\section{Introduction}

Surface atoms in covalently bound crystals reorganize, forming surface reconstructions, in order to minimize the energy due to the large number of dangling bonds created when the surface is cleaved. The structure of the resulting surface reconstructions is governed by (1) local chemistry, which drives the formation of new covalent bonds to minimize energy, (2) long range electrostatics, which require the surface to remain charge neutral [1], and (3) local displacement strain, which increases the energy as the new bonds require atoms to be displaced from their bulk atomic positions. A variety of different surface reconstructions can be obtained for a single material system by altering the growth conditions, specifically the impinging flux and temperature. In the case of compound semiconductors, such as the III V or
II VI semiconductors, the overpressure of the more volatile anion will also alter the local surface chemistry, impacting the surface reconstruction. The surface reconstructions of homoepitaxial films are generally well understood through a combination of experiment and density functional theory (DFT) studies. GaAs exhibits a $\mathrm{c}(4 \times 4), \beta 2(2 \times 4)$ and a $\zeta(4 \times 2)$ reconstruction with decreasing As chemical potential, $\mu_{\mathrm{As}}$, [2], and GaSb exhibits an $\alpha, \beta$ and $\gamma(4 \times 3)$ reconstruction followed by a $\mathrm{c}(2 \times 10)$ reconstruction with increasing $\mathrm{Sb}$ chemical potential, $\mu_{\mathrm{Sb}}$, although the details of the $\mathrm{c}(2 \times 10)$ reconstruction are not well understood $[3,4]$. While the three competing interactions described above describe the physics governing homoeptiaxial surface reconstructions, the reconstructions of heteroepitaxial surfaces are further impacted by atomic size mismatch strain determined by the local chemistry of alloyed films [5], and by lattice mismatch strain that may stabilize reconstructions not seen at the relaxed lattice parameter [6]. 

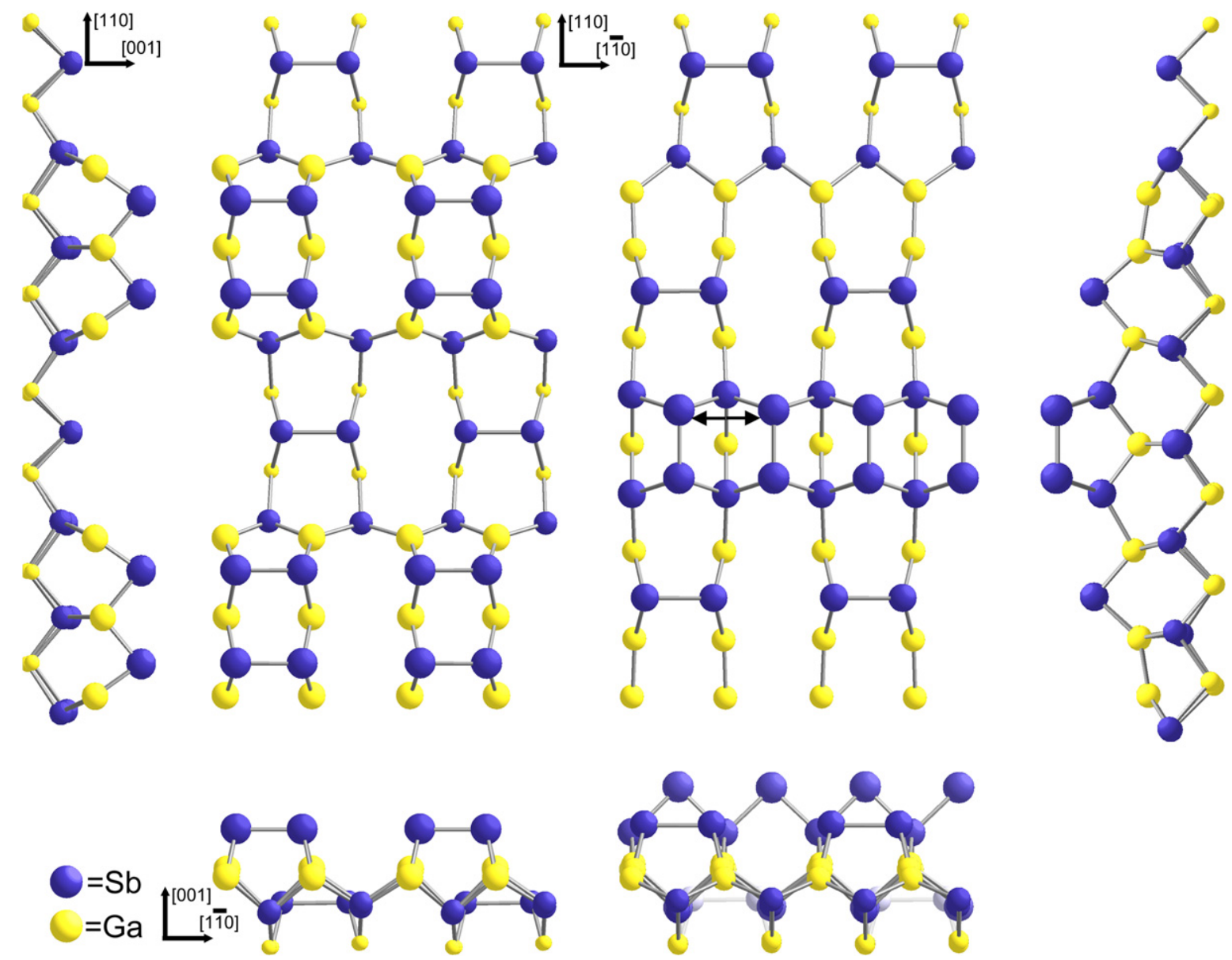

Figure 1. Schematic surface atomic structures of the (left) $\beta 2(2 \times 4)$ and (right) $\alpha(2 \times 8)$ reconstructions. Some atoms removed for clarity. Arrows in the $(2 \times 8)$ reconstruction show the dimer backbond connecting adjacent unit cells.

Understanding the physics governing surface reconstructions is important in epitaxial film growth because of the dramatic impact of surface structures on subsequent film growth. The surface reconstruction has been demonstrated to impact interfacial development [7], catalysis [8], oxide growth [9], atomic bulk ordering [7], epitaxial film growth [10], and magnetic domain orientation [11]. Different studies have examined how atoms diffuse on a reconstructed surface [12] and how atoms are incorporated into the growing film [13]. It has also been shown that films grown on the same substrate under different growth conditions can have dramatically different defect structures. For example, the growth of $\mathrm{SiGe} / \mathrm{Si}$ results in a high number of threading dislocations due to the high lattice mismatch of these films, reducing the electron diffusion length in these films. However, by changing the growth conditions to a layer-by-layer growth, the surface structure is altered, resulting in a mixture of $\mathrm{Si} / \mathrm{Ge}$ at the interface and reducing the strain and introducing edge defects [14]. Another example is in low temperature GaAs (LT GaAs) growth, where the amount of As introduced into the system affects the precipitation of As particles dramatically impacting the dislocation density in these films [15]. However, it remains unclear whether the different defect structures are due to differences in the growth kinetics alone or due to differences in the surface reconstruction of the substrate upon which the film is grown.

This paper examines the role of the surface reconstruction on subsequent film growth and defect nucleation in heteroepitaxial $\mathrm{GaSb} / \mathrm{GaAs}(001)$ films. Two films were grown under the same conditions (growth rate, temperature, $\mathrm{Sb}$ overpressure) on different initial surface reconstructions in order to separate the effects of film growth kinetics from those of the atomic surface structure. The resulting films show dramatic differences in surface topography, strain relaxation and defect structure, demonstrating the impact of the surface reconstruction and initial atomic incorporation on the defect structure of heteroepitaxially grown thin films.

\section{Experimental methods}

Samples were grown on an EPI930 MBE chamber with valved, solid source $\mathrm{Ga}, \mathrm{Sb}$ and As at temperatures determined by an optical pyrometer. Growth rates were determined through reflection high energy electron diffraction (RHEED) oscillations, and samples were characterized in situ with RHEED and multi-beam optical stress sensor (MOS) measurements and ex situ with atomic force microscopy (AFM) and transmission electron microscopy (TEM). Samples were 


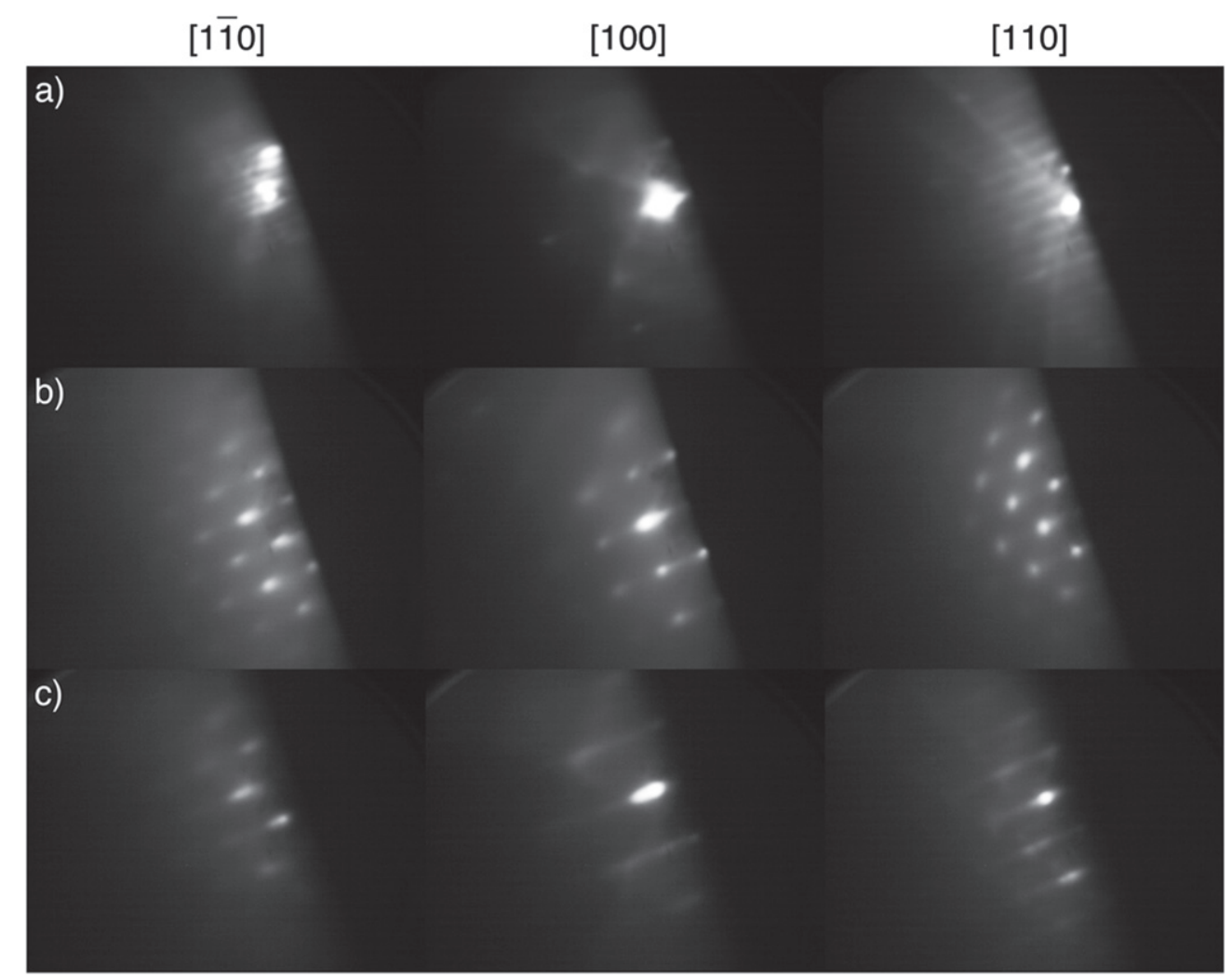

Figure 2. RHEED images taken (a) after buffer deposition (b) after deposition of $100 \mathrm{ML} \mathrm{GaSb}$ on the (2×4) reconstruction and (c) after deposition of $100 \mathrm{ML} \mathrm{GaSb}$ on the $(2 \times 8)$ reconstruction.

grown on $150 \mu \mathrm{m}$ thick wafers freely mounted in Mo rings to allow characterization of the stress evolution of the sample by MOS. The surface oxide was desorbed at $600 \leqslant T \leqslant 625^{\circ} \mathrm{C}$ and a $0.5 \mu \mathrm{m}$ buffer was grown at $600{ }^{\circ} \mathrm{C}$ at $R_{\mathrm{GaAs}} \sim 1.2 \mathrm{ML} \mathrm{s}^{-1}$. At this point the surface shows a $(2 \times 4)$ reconstruction according to RHEED. Two starting surface reconstructions were examined: the As-rich $(2 \times 4)$ reconstruction, and the Sb-rich $(2 \times 8)$ reconstruction. The GaAs $(2 \times 4)$ reconstruction is present on the surface as a result of the substrate preparation, thus the sample temperature is simply lowered to the growth temperature of $T=525^{\circ} \mathrm{C}$ under an $\mathrm{As}_{4}$ overpressure. The $(2 \times 8)$ surface was prepared by closing the As valve while still at the buffer growth temperature of $T=600{ }^{\circ} \mathrm{C}$. The excess $\mathrm{As}_{4}$ in the chamber was pumped out, and As was allowed to desorb from the sample surface resulting in a surface reconstruction change to the Ga rich $(4 \times 2)$. The $\mathrm{Sb}_{4}$ valve and shutter were then opened and the sample was lowered to the growth temperature, $T=525^{\circ} \mathrm{C}$, resulting in a Sb-rich $(2 \times 8)$ reconstruction according to RHEED. GaSb films were grown on both surfaces at $R_{\mathrm{GaSb}} \sim 0.37 \mathrm{ML} \mathrm{s}^{-1}$ with an $\sim 1: 1 \mathrm{~V}:$ III ratio to thickness of $h=100$ monolayers (MLs). Each sample was produced using the same growth temperature, growth rate, and $\mathrm{Sb}$ flux while only varying the initial atomic surface structure. This allows any differences between the samples to be attributed to the difference in starting surface structure rather than changes in temperature or $\mathrm{V}$ : III ratio.

\section{Results and discussion}

In order to examine the effect of surface reconstruction on film growth and defect formation, two samples were grown under the same growth parameters but on two different starting surface reconstructions: the $\beta 2(2 \times 4)$ and the $\alpha(2 \times 8)$ reconstruction. The $\beta 2(2 \times 4)$ reconstruction is well known for homoepitaxial GaAs and has been well characterized [16]. The $\alpha(2 \times 8)$ reconstruction is stable for the strained system of Sb-capped GaAs [17 19]. Schematic atomic structures of these two reconstructions along three different crystallographic axes are shown in figure 1 . There are two variants of the $(2 \times 4)$ and $(2 \times 8)$ reconstructions, named alpha and beta. The difference between them is the presence of one or two surface As dimers, respectively, which are located adjacent to the trench dimer. In the case of the $(2 \times 4)$ reconstruction, the alpha structure is not thermodynamically stable for $\mathrm{GaAs}(001)$ [2], although it has been seen under some experimental conditions. In the case of the $(2 \times 8)$ reconstruction, DFT results suggest that the thermodynamically stable surface reconstruction is the $\alpha(2 \times 8)$ [17] rather than the $\beta(2 \times 8)$.

The GaSb films grown on both starting reconstructions were characterized during growth by RHEED, which shows an immediate difference in the surfaces that develop. Figure 2 shows RHEED images of the [110], [001] and [110] directions of a sample upon completion of the buffer layer (top), and after growth of $100 \mathrm{ML} \mathrm{GaSb}$ on the $(2 \times 4)$ (middle) and 


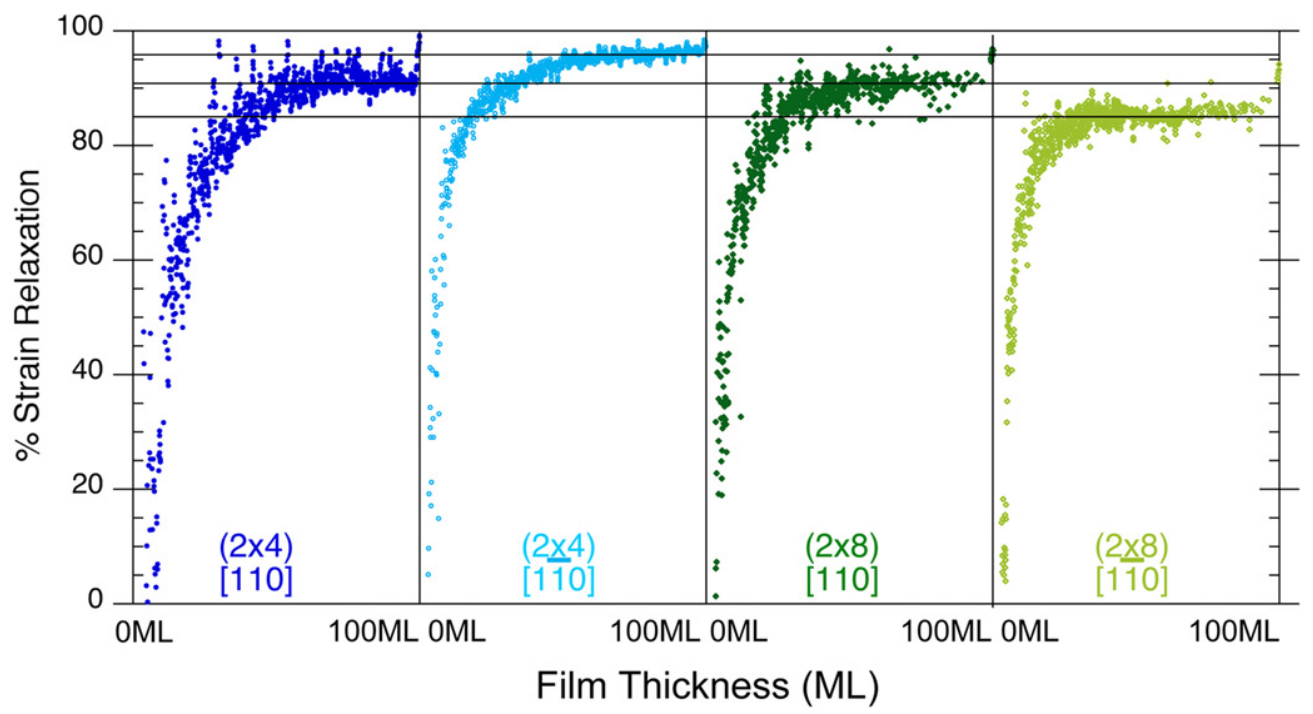

Figure 3. Percent strain relaxation of 100 ML GaSb grown on a $(2 \times 4)$ and $(2 \times 8)$ reconstruction. The width of each box is $100 \mathrm{ML}$. The black lines are guides for the eye to show the maximal strain relaxation of each sample.

$(2 \times 8)$ reconstructions (bottom). The initial $(2 \times 4)$ and $(2 \times 8)$ surface reconstructions appear streaky in the RHEED pattern and previous scanning tunneling microscopy characterization of both the $(2 \times 4)$ and $(2 \times 8)$ surfaces $[16,17]$ shows these RHEED patterns correspond to flat surfaces with large, reconstructed terraces. During GaSb deposition, the intensity of the reconstruction streaks in the RHEED pattern decreases indicating that the disorder on the surface is increased on both the $(2 \times 4)$ and $(2 \times 8)$ samples. The film grown on the $(2 \times 4)$ reconstruction has a spotty RHEED pattern in both $<110>$ indicating surface roughening, while the $(2 \times 8)$ sample retains a somewhat streaky pattern along the [001] and [110] directions indicative of a slightly smoother surface.

The films were also characterized during growth by MOS, an in situ characterization tool that measures changes in wafer curvature during film deposition. The curvature, which is directly related to the sample strain through Stoney's equation [20], is measured in situ by examining the change in spot spacing of an array of parallel laser beams reflected from the sample surface. MOS data was collected during formation of the $(2 \times 8)$ reconstruction and during film deposition on the $(2 \times 4)$ and $(2 \times 8)$ surfaces. No change in wafer curvature was recorded during formation of the $(2 \times 8)$ surface because the thickness resolution in our MOS set-up is on the order of $10 \AA$. Deposition of $100 \mathrm{ML}$ of GaSb on both the $(2 \times 4)$ and $(2 \times 8)$ reconstructions introduces a significant amount of strain. Figure 3 displays percentage of strain relaxation, $\gamma$, versus film thickness, assuming 100\% incorporation of deposited material. There are three growth regimes for films grown on the $(2 \times 4)$ and $(2 \times 8)$ reconstructions: an initial fast relaxation of strain, a transition with a decreasing slope, and a plateau where most of the strain has been relieved and the remaining strain relaxation remains constant. The curves for the films grown on the $(2 \times 4)$ reconstruction are very distinct from one another along the two $<110>$ directions, indicating that the surface symmetry differences in the two directions directly impact the strain relaxation of the surface. The initial slopes of the two curves are slightly different, with the [110] displaying a slower strain relaxation. Fitting the initial linear portion of the strain relaxation curves results in slopes of $2.1 \% / \mathrm{ML}$ and $2.5 \% / \mathrm{ML}$ in the [110] and [110], respectively. Both curves plateau at approximately the same time, $200 \mathrm{~s}$, corresponding to $60 \mathrm{ML}$. The [110] relieves $91 \%$ of the strain, while the [1ㅣㅣ relieves $96 \%$, consistent with the difference in the initial slopes of the strain relaxation curves. The curves for the film grown on the $(2 \times 8)$ reconstruction are somewhat different, showing the same initial relaxation rate for both $<110>$, $\sim 3.3 \% / \mathrm{ML}$, faster than the $(2 \times 4)$ sample. The relaxation plateaus are at slightly different points in the growth, with the $[1 \overline{1} 0]$ reaching the plateau region at $\sim 30 \mathrm{ML}$, and the [110] reaching the plateau region at $\sim 40$ ML. Because of the different plateau points, the [110] relieves more strain than the $[1 \overline{1} 0]$ at $92 \%$ and $85 \%$, respectively.

The MOS profiles measure the kinetics of the strain relaxation of the surface. The data shows that the film grown on the $(2 \times 4)$ reconstruction exhibits more absolute strain relaxation than the film grown on the $(2 \times 8)$ reconstruction. However, the $(2 \times 4)$ film requires more thickness to reach the maximum relaxation, whereas the $(2 \times 8)$ film relieves less of the total strain, but does so earlier in the growth. These differences in the amount of strain relieved and the amount of deposited film needed to relieve the strain suggests that there are distinct differences between how strain is relaxed within the film on the $(2 \times 4)$ and $(2 \times 8)$ surfaces, perhaps due to differences in the diffusion of $\mathrm{Ga}$ and $\mathrm{Sb}$ on these surfaces, or due to the injection of strain relieving defects at the $\mathrm{GaSb}$ / GaAs interface.

The difference in strain relaxation along the [110] is initially surprising, since both the $(2 \times 4)$ and $(2 \times 8)$ reconstructions exhibit a periodicity that is twice that of the bulk lattice parameter along this direction. However re-examination of the atomic structure (figure 1) reveals an important difference. The $(2 \times 8)$ structure has a straight row of 

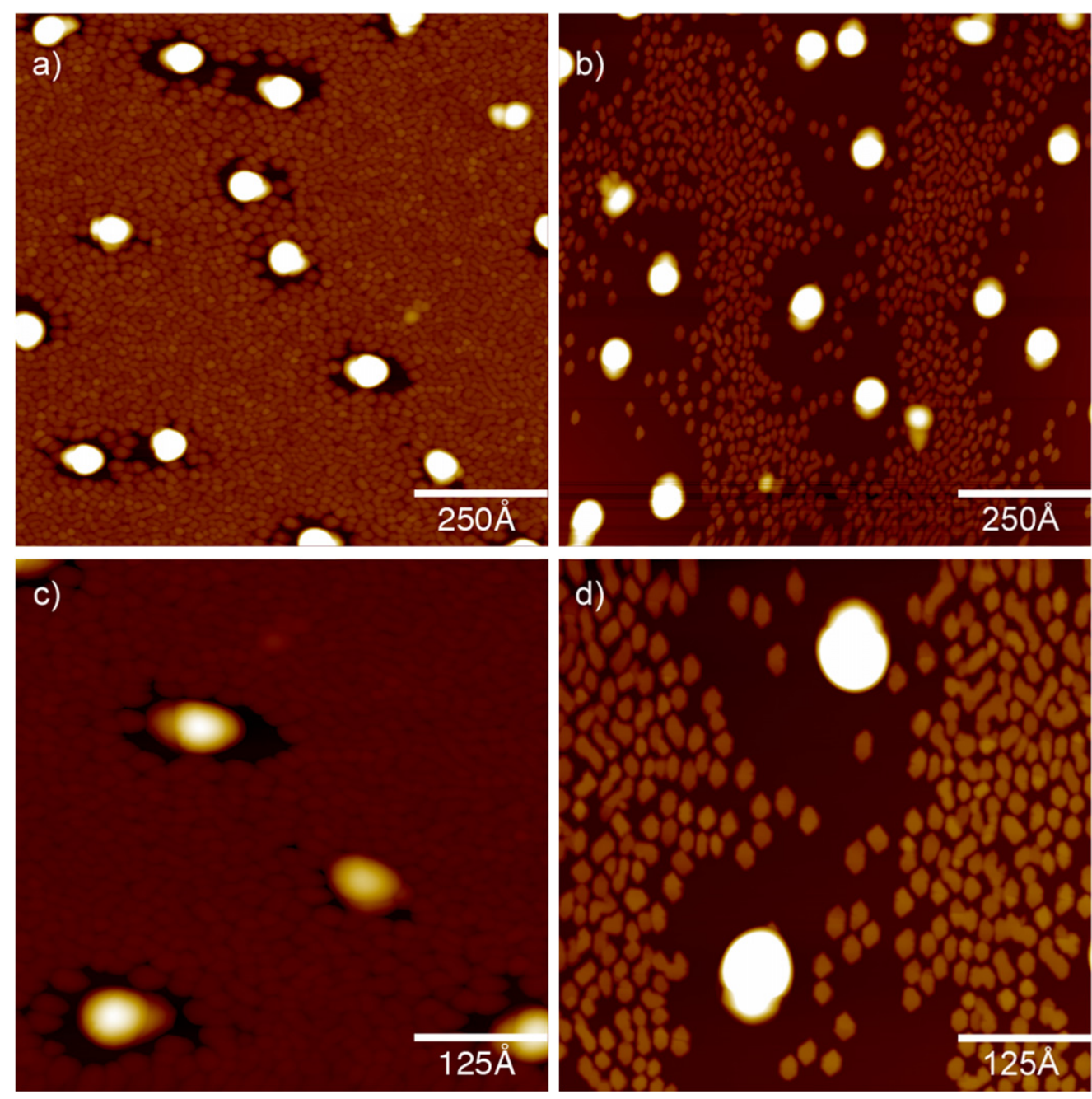

Figure 4. AFM images of $100 \mathrm{ML} \mathrm{GaSb/GaAs} \mathrm{grown} \mathrm{on} \mathrm{(a)} \mathrm{and} \mathrm{(c)} \mathrm{the}(2 \times 4)$ reconstruction and (b) and (d) the $(2 \times 8)$ reconstruction. (top) $10 \mu \mathrm{m}$ images and (bottom) $5 \mu \mathrm{m}$ images.

connected anion dimers that link adjacent unit cells along the [11̄0] through a series of covalent bonds (indicated by the arrows in figure 1). The connected dimer row limits the amount of compression/tension that can be accommodated along the [110]. In the $(2 \times 4)$ reconstruction, adjacent cells are not connected by such row of dimers, allowing this surface reconstruction to accommodate more deformation along the [110]. Work by Thomas et al shows that the two dimers in the $\beta 2(2 \times 4)$ reconstruction bow outwards in order to accommodate strain when a larger atom is placed in the center of the row [21]. The lack of fixed dimer bonds along the [11̄0] in the $(2 \times 4)$ reconstruction may allow a similar distortion within the lattice to accommodate strain, and/or allow for the injection of strain relieving dislocations. In either case, the formation of the $\mathrm{GaSb} / \mathrm{GaAs}$ interface, and with it the formation of different types of interfacial defects, likely dictates the final strain relaxation of the films.

The final surface morphology of the films grown on the $(2 \times 4)$ and $(2 \times 8)$ surfaces are significantly different, as seen in the AFM micrographs in figure 4. The films grown on either surface consist of a bimodal distribution of large and small islands. The large islands are very similar between the two samples. They are approximately round with basal areas of $350 \times 10^{3} \pm 70 \times 10^{3} \mathrm{~nm}^{2}$ and $390 \times 10^{3} \pm 80 \times 10^{3} \mathrm{~nm}^{2}$ for the $(2 \times 4)$ and $(2 \times 8)$ samples, respectively. The densities of the large islands are also very similar between the two samples at $1.43 \times 10^{7} \mathrm{~cm}^{-2}$ and $1.75 \times 10^{7} \mathrm{~cm}^{-2}$ for the $(2 \times 4)$ and $(2 \times 8)$ samples respectively. It should be noted that the large islands in both samples often have a second island that is adjacent to the large islands. These second islands appear on multiple sides of the large islands, and are confirmed by TEM, eliminating the possibility that they are artifacts of AFM imaging. For statistical determinations, these double dots have been treated as a single feature due to the difficulty in accurately determining the height and area of the smaller one, resulting in a slight overestimation of the large island area. For both samples, there is also a denuded area around the large islands. The larger denuded area around the islands in the $(2 \times 8)$ sample suggests that diffusion is faster on this surface.

The dramatic topographical difference between these two samples is manifested in the shape, size, and density of the smaller dots. On the $(2 \times 4)$ sample, the small dots are very 


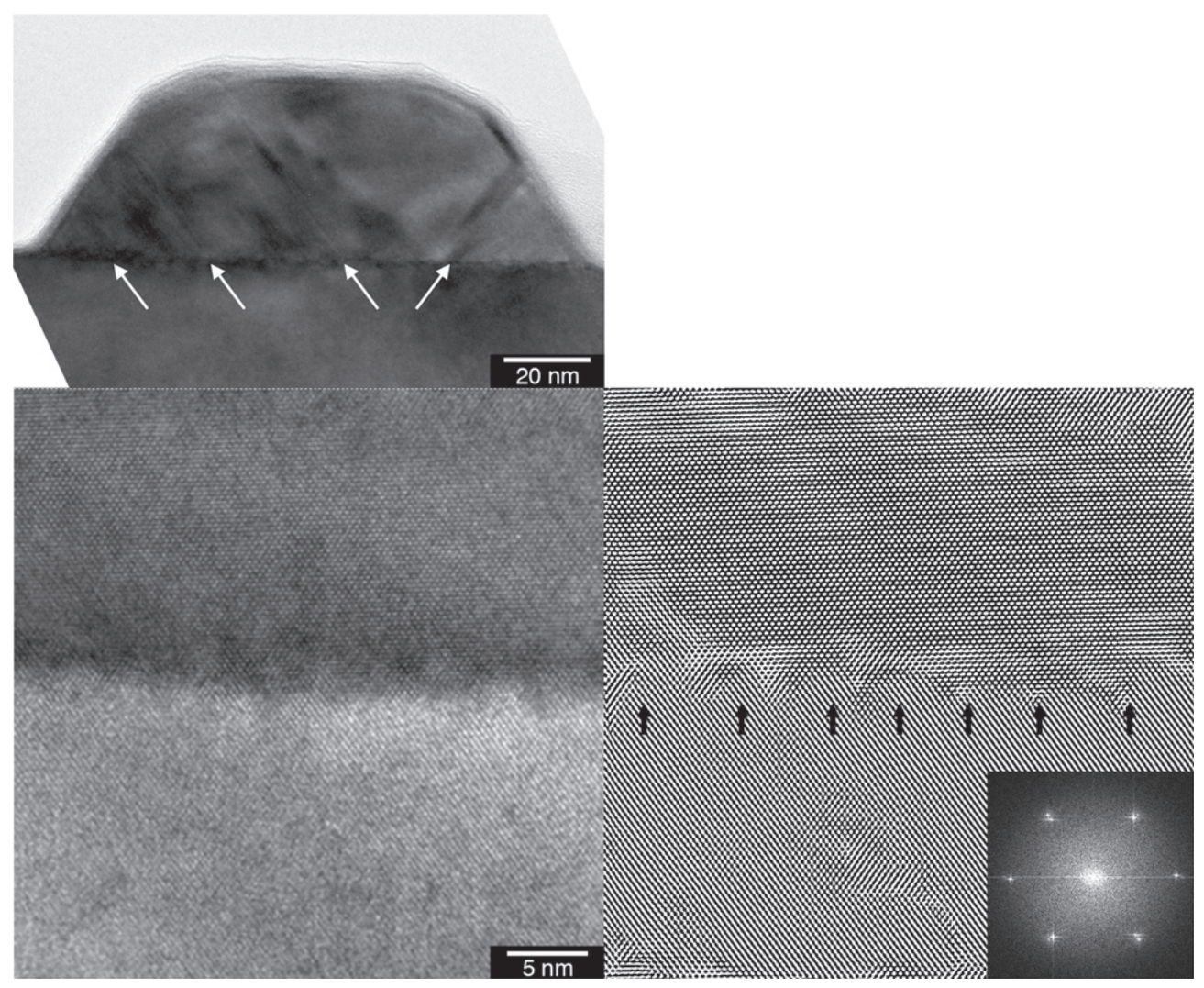

Figure 5. (top) Bright field TEM image of stacking faults and (bottom) TEM image and inverse FFT TEM image of edge dislocations grown on the $(2 \times 4)$ sample. The FFT of the original HRTEM image is inset in the lower right corner of the filtered image.

densely packed and tend to agglomerate into chains. The density of the small dots is $3.26 \times 10^{9} \mathrm{~cm}^{-2}$, and they cover $\sim 89 \%$ of the sample surface, compared to the $5 \%$ of the surface covered by the large islands. The small islands grown on the $(2 \times 8)$ surface are almost three times smaller than for the $(2 \times 4)$ surface, at only $1.09 \times 10^{9} \mathrm{~cm}^{-2}$, resulting in a lower surface coverage. In addition, the dots resemble hexagons elongated along the [1 $1 \overline{1} 0]$, with points forming at the tips oriented along the [110]. The elongation and the formation of facets in these islands suggest that diffusion is faster along the [110] in the $(2 \times 8)$ sample, consistent with experimental observations in other materials systems [22]. This is also in agreement with the fact that the denuded zone around the large islands appears longer along the [110] than the [110]. If the denuded zone is characterized by an ellipse that extends with radii in the $\langle 110\rangle$ directions until a small dot is touched, it is apparent that the diffusion length along the [110] is approximately twice that of the diffusion length in the [110]. This in agreement with the fact that in $(2 \times 4)$ reconstructions the primary direction of diffusion is along the row of dimers in the [110].

Further analysis of the films grown on the $(2 \times 4)$ and $(2 \times 8)$ reconstructions shows that the differences in these films go beyond surface topography and that the islands also have different defect structures, as expected from the different relaxation profiles measured in the MOS. Bright-field TEM imaging shows that the $(2 \times 4)$ dots exhibits two defect structures, stacking faults and edge dislocations, both of which can be seen in the cross sectional TEM images shown in figure 5. The edge dislocations often consist of two $1 / 2$ planes of atoms that terminate at the boundary between the film and substrate. The extra planes extend into the GaAs substrate, as is expected due to the larger lattice parameter of $\mathrm{GaSb}$. The dislocations are spaced at $\sim 15$ lattice planes apart.

The defect structures for the film grown on the $(2 \times 8)$ reconstruction are edge dislocations and a tilt boundary. This can be seen in analysis of the diffraction pattern, as well as some of the FFT patterns, which show that the additional spots are rotated relative to those of the substrate. This tilt boundary is also visible in TEM images such as the one shown in figure 6 . The top image shows a $5^{\circ}$ tilt boundary which, together with the array of dislocations visible in the lower image, relieves the strain. In this case, the tilt disappears when the dislocations appear and vice versa. The dislocations have no apparent regularity in their spacing in the obtained micrographs.

The TEM results coupled with the MOS, RHEED and AFM data show that there is a distinct difference in the surface topography, defect structure, and strain relaxation between samples grown on a $(2 \times 4)$ and $(2 \times 8)$ reconstruction surface. This demonstrates that the initial incorporation of atoms onto the surface greatly impacts the film development. Previous work has demonstrated that in $\mathrm{GaSb} / \mathrm{GaAs}$ the growth parameters, including flux and temperature, result in either an array of misfit dislocations or the nucleation of 


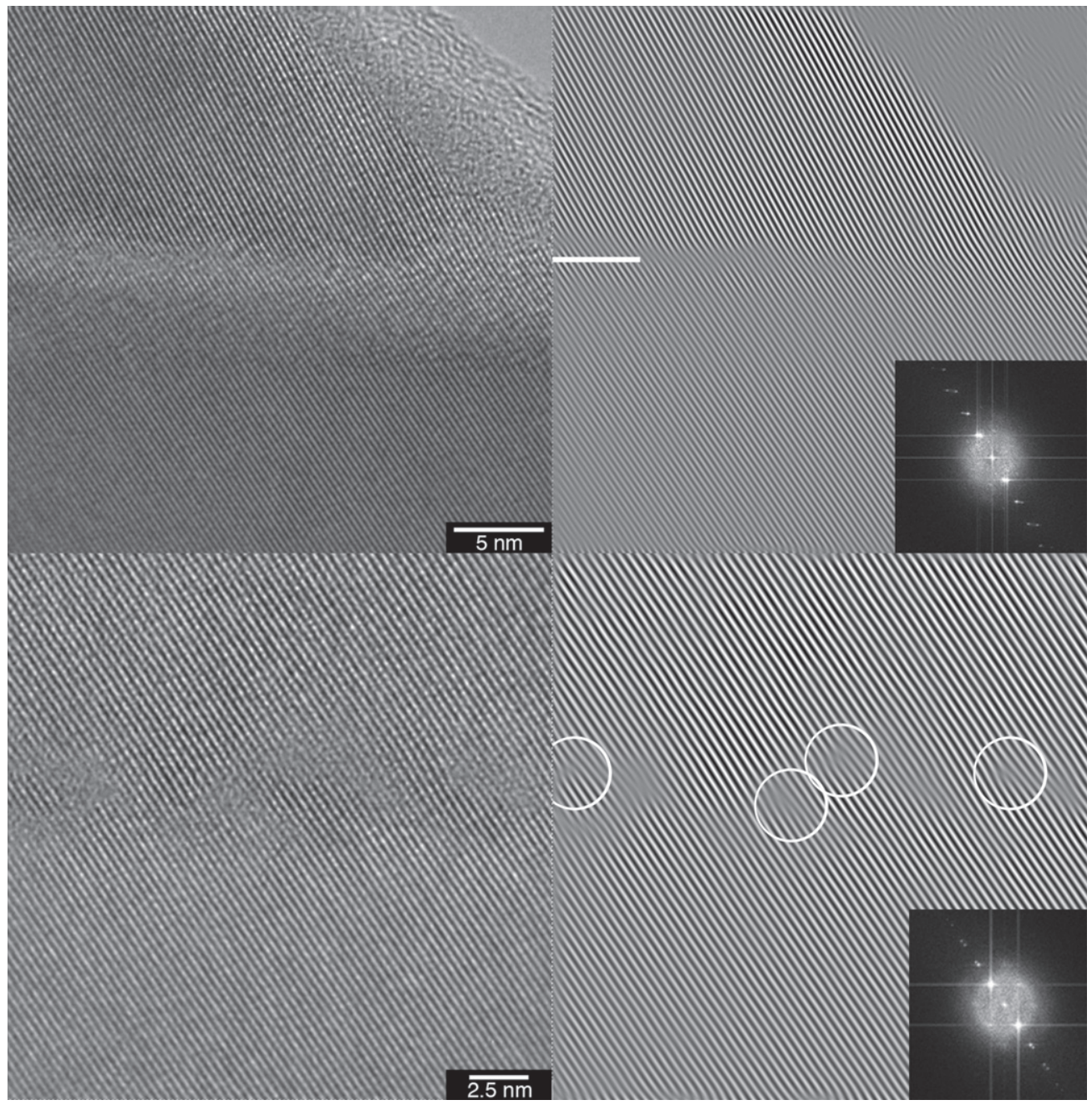

Figure 6. Bright field TEM and inverse FFT TEM images of (top) $5^{\circ}$ tilt boundary and (bottom) dislocations from $(2 \times 8)$ sample. The FFTs of the original HRTEM images are inset in the lower right corner of the filtered images.

threading dislocations [23 27]. Similar growth parameters were used in both our study and these studies but resulted in different defect structures. This could be due to variations in the growth conditions such as slight variations in the temperature, the use of Group V dimers versus tetramers, small variations in V/III ratio, or even small, unintentional variations in wafer miscut. Regardless, the different relaxation mechanisms shown in both this work and previous reports demonstrate the range of strain relaxation mechanisms possible ranging from unequally spaced dislocations to dislocation arrays and tilt boundaries. While previous work has found that the surface reconstruction can affect bulk ordering by affecting how atoms are incorporated into the film [7], to the best of our knowledge this is the first demonstration that the surface reconstruction can affect or even control the nucleation of defect structures. By focusing on the differences that result when an identical film is grown on two different starting surfaces, this work shows that atomic incorporation and defect nucleation at interfaces is dramatically impacted not only by growth conditions, but also by the starting surface reconstruction.

\section{Conclusions}

The work described shows the dramatic impact of the starting surface on film growth. Films grown on either the $(2 \times 4)$ or $(2 \times 8)$ exhibit dramatic differences in strain relaxation of the film, topography, and defect nucleation. Films grown on the $(2 \times 4)$ surface show significant relaxation differences along the [110] and [110]. The resulting islands have a bimodal size distribution, with large islands closely surrounded by smaller round islands. TEM results show that relaxation of the islands occurs through the injection of edge dislocations. The $(2 \times 8)$ surface relieves less strain than the $(2 \times 4)$ but relieves it faster during growth. The [110] relaxes more due to the ability of the surface dimers to bow out to accommodate the strain. The resulting morphology also exhibits a bimodal distribution of islands but with a smaller density of the small hexagonal shaped islands. TEM results show that the large islands on this surface relax by a $5^{\circ}$ tilt boundary. These results demonstrate the possibility to engineer specific defects into films by controlling the starting surface of film growth and demonstrate that the surface reconstruction may actually 
control the nucleation of specific strain relaxation mechanisms.

\section{Acknowledgements}

The authors thank K Grossklaus for his help with TEM characterization and $\mathrm{C}$ Pearson for interesting discussions. We also acknowledge funding from DOE/BES (ER46172). JMM gratefully acknowledge Chakrapani Varanasi and the support of the Department of Defense, Army Research Office via the Grant No. W911NF-12-1-0338.

\section{References}

[1] Pashley M D 1989 Phys. Rev. B 4010481

[2] Thomas J C, Modine N A, Millunchick J M and Van der Ven A 2010 Phys. Rev. B 82165434

[3] Tinkham B P, Romanyuk O, Braun W, Ploog K H, Grosse F, Takahasi M, Kaizu T and Mizuki J 2008 J. Electron. Mater. 3717938

[4] Houze J, Kim S, Kim S G, Erwin S C and Whitman L J 2007 Phys. Rev. B 76205303

[5] Bickel J E, Van der Ven A, Millunchick J M and Modine N A 2008 Appl. Phys. Lett. 92062104

[6] Bickel J E, Modine N A, Pearson C and Millunchick J M 2008 Phys. Rev. B 77125308

[7] Froyen S and Zunger A 1996 Phys. Rev. B 534570

[8] Somorjai G A 1994 Ann. Rev. Phys. Chem. 45721

[9] Zhou G and Yang J C 2005 J. Mater. Res. 20168494

[10] Voigtländer B 2001 Surf. Sci. Rep. 43127

[11] Welp U, Vlasko Vlasov V K, Liu X, Furdyna J K and Wojtowicz T 2003 Phys. Rev. Lett. 90167206
[12] Wixom R R, Rieth L W and Stringfellow G B 2004 J. Cryst. Growth 26536774

[13] Kratzer P, Penev E and Scheffler M 2003 Appl. Surf. Sci. 216 43646

[14] Sakai A, Taoka N, Nakatsuka O, Zaima S and Yasuda Y 2005 Appl. Phys. Lett. 86221916

[15] Chang M N, Hsieh K C, Nee T E and Chyi J I 1999 J. Appl. Phys. 862442

[16] Ratsch C, Barvosa Carter W, Grosse F, Owen J H G and Zinck J J 2000 Phys. Rev. B 12 R7719

[17] Bickel J E, Modine N A and Millunchick J M 2009 Surf. Sci. $\mathbf{6 0 3} 29459$

[18] Whitman L J, Bennett B R, Kneedler E M, Jonker B T and Shanabrook B V 1999 Surf. Sci. Lett. 436 L707 14

[19] Laukkanen P, Perala R E, Vaara R L, Vayrynen I J, Kuzmin M and Sadowski J 2004 Phys. Rev. B 69205323

[20] Floro J A and Chason E 1996 Appl. Phys. Lett. 693830

[21] Thomas J C, Millunchick J M, Modine N A and Van der Ven A 2009 Phys. Rev. B 80125315

[22] Rosini M, Righi M C, Kratzer P and Magri R 2009 Phys. Rev. B 79095302

[23] Balakrishnan G, Rotter T J, Jallipalli A, Dawson L R and Huffaker D L 2008 Solid State Lasers XVII: Tech. Devices $\mathbf{6 8 7 1} 687111$

[24] Balakrishnan G, Tatebayashi J, Khoshakhlagh A, Huang S H, Jallipalli A, Dawson L R and Huffaker D L 2006 Appl. Phys. Lett. 89161104

[25] Huang S H, Balakrishnan G, Khoshakhlagh A, Jallipalli A, Dawson L R and Huffaker D L 2006 Appl. Phys. Lett. 88 131911

[26] Jallipalli A, Balakrishnan G, Huang S H, Khoshakhlagh A, Dawson L R and Huffaker D L 2007 J. Cryst. Growth 303 44955

[27] Tatebayashi J, Khoshakhlagh A, Huang S H, Dawson L R, Balakrishnan G and Huffaker D L 2006 Appl. Phys. Lett. 89 203116 